\title{
COMPARATIVE STUDY ON ENERGY CONSUMPTION IN DYNAMIC WINDOW SECURED IMPLICIT GEOGRAPHIC FORWARDING ROUTING PROTOCOL
}

\author{
${ }^{1}$ Abubakar Idris Umar and ${ }^{2}$ Zurina Mohd Hanapi \\ ${ }^{1}$ Department of Computer Science, Kano University of Science and Technology, Kano, Nigeria \\ ${ }^{2}$ Department of Computer Networks, University Putra Malaysia, Selangor, Malaysia
}

Received 2014-01-03; Revised 2014-01-13; Accepted 2014-04-09

\begin{abstract}
An Ideal WSNs should operate with the least possible energy required in order to increase the lifetime of the sensor nodes and at the same time, ensure network connectivity. But the Inherent power limitation makes power-awareness a critical requirement for WSN, this calls for the need to manage energy in sensor nodes. Also In order to ensure successful transmission of data from sensor node source to destination, it becomes necessary to maintain network availability. The network must be resilient to individual node failure which can happen due to zero power posses by the node and due to security attacks posed on the node and the network. Dynamic Window Secured Implicit Geographic Forwarding (DWSIGF) routing protocol has proven to be robust, efficient and resistant to some security attack which causes failure in network availability. However the extent to which energy is consumed in sensor nodes which deploys DWSIGF as its routing protocol has never been mentioned. In this research, we performed a comparative study on energy consumption in DWSIGF routing protocol. Using the first order radio model, we determined the energy consumed in a network. The protocol (DWSIGF) is matched up against its counterpart SIGF as the traffic is increased. Observation shows that DWSIGF due to the variable timing assigned to the CTS collection window, CTS signal fails to reach destination as collection window time expires, thus the need for retransmission. This in turn consumes more energy than the counterpart SIGF which has a fixed CTS collection time. The simulation work was done using Matlab 7.0. Energy consumed in the random variant of both protocols (DWSIGF and SIGF) was also observed to be higher than the priority variant of the protocols.
\end{abstract}

Keywords: DWSIGF, SIGF, Energy Consumption, Handshaking, MAC

\section{INTRODUCTION}

The most important features of a sensor are its size, low cost and low energy consumption which varies from application to application. These features made Wireless Sensor Network (WSN) a potential focus of many research efforts, both in academia and industry (Akyildiz et al., 2002; 2007; Bulusu et al., 2000). In WSN, the sensor nodes have to act both as data processing nodes and communication nodes which is very much different from the traditional network, this makes the routing design of a protocol a bit more demanding due to the limitations suffered by the nodes; easily destroyed, exhausted of energy or power, lower bandwidth, little processing power and limited sensing region (Stankovic, 2004). In order to ensure successful transmission of data from sensor node's source to destination, it becomes necessary to maintain network availability (Al-Karaki and Kamal, 2004).

The network must be resilient to individual node failure. This Node failure can happen due to zero power energy posses by the node and due to security attacks posed on the node and the network (Hanapi et al., 2009).

In a multi-hop wireless sensor network, each node plays the dual role of data originator (source or Kano, Nigeria 
generator) and data router. The failure of even few sensor nodes can cause significant topological changes and might require rerouting of the packets and reorganization of the network. Hence, energy conservation and power management mechanisms have to be given additional significance in WSN (Gomez et al., 1999; Stankovic, 2004).

A Medium Access Control protocol (MAC) coordinates the actions of communication in a WSN (Stankovic, 2006). An effective MAC protocol for WSN must consume little power, avoid collisions, be implemented with a small code size and memory requirements, be efficient for a single application and be tolerant to changing frequency and networking conditions (Stankovic, 2006; He et al., 2007). In this study we considered the IEEE 802.11 DCF (fundamental MAC technique). In IEEE 802.11, carrier sensing is performed at both the air interface, referred to as physical carrier sensing and at the MAC sub layer, referred to as virtual carrier sensing. The virtual carrier sense mechanism provides the capability of exchanging short signals such as the Request To Send (RTS), Clear To Send (CTS) and Acknowledgment (ACK). Some protocols designed in WSN make use of this feature to improve on the connectivity and reliability of WSN's protocols. This can be found in protocols such as Implicit Geographic Forwarding (IGF), Energy Efficient MAC Protocol (EEMP) and Angled transmission, Back-off relay and Cancellation (ABC) protocol. This is made possible because the DCF function is devoid of the sleep mode. However, it is important to note that the reliability of the MAC comes at a price in terms of energy consumption and delay, even though the design of a geographic routing protocol is to minimize the MAC layer cost involved which is associated to energy consumption (Lee et al., 2008).

In this study, we performed a comparative study on energy consumption in Dynamic Window Secured Implicit Geographic Forwarding Protocol (DWSIGF) protocol (A protocol derived from IGF and enhanced with security features). Using the first order radio model proposed by Heinzelman et al. (2000), we determined the energy consumed in a network while using the DWSIGF as a routing protocol. The protocol (DWSIGF) was matched up against its counterpart Secured Implicit Geographic Protocol (SIGF) by Wood et al. (2006) as the traffic increases. Observation shows that in DWSIGF, due to the variable timing assigned to the CTS collection window, CTS signal fails to reach destination as collection window time expires, thus the need for retransmission. This in turn consumes more energy than the counterpart SIGF which has a fixed CTS collection time. The simulation work for this was done using Matlab 7.0. Random variant of both protocols (DWSIGF and SIGF) was also observed to be higher than the priority variant of the protocols.

\section{RELATED WORKS}

\subsection{DWSIGF and SIGF}

The two protocols (DWSIGF and SIGF) originated from IGF. IGF is a combined routing/MAC protocol that assumes nodes have knowledge of their location (and optionally remaining energy) to make non deterministic forwarding decisions when routing pointto-point traffic (Blum et al., 2003; Hanapi et al., 2009). It uses the concept of lazy binding to establish the stateless property. Thus when a packet is being transmitted, the next forwarding node is not known to the transmitting node, the receiving node will therefore have to contend with each other in respond to the transmitting node (Lee et al., 2008; Blum et al., 2003). This stateless property allows it handle network dynamics effortlessly since it does not have a routing table to make forwarding decisions with.

SIGF protocol by Wood et al. (2006) has the same property as the IGF protocol but with improved good enough security and high performance. Wood et al. (2006) shows that SIGF is capable of preventing many common attacks against routing. However, there is a tradeoff between security provided by the protocol and efficiency since sensors are regarded as resource constrained devices and have limitation in memory and 3 processing power. SIGF like IGF employs the handshaking process for communication. The process is initiated upon the transmission of an Open Request to send Signal (ORTS) which is broadcasted. Neighbors within the broadcast range $\left(60^{\circ}\right.$ sextant) considered eligible to forward the message. On receiving the signal, A CTS response timer is initiated which on expiry, a CTS packet is sent and the data is transferred from the ORTS sender in a DATA message after which an ACK signal is sent to acknowledge the received data (Hanapi et al., 2009; Wood et al., 2006). This same sequence of operation is performed by DWSIGF communication process.

The valid duration of the CTS timer provided is called the CTS response window. SIGF provided a fixed time of $5 \mathrm{~ms}$ because a real time devices such as a sensor is suppose to respond predictably. This made the 
protocol less vulnerable to CTS rushing attack. Since the attacker now knows the duration for which the window for a response was open. DWSIGF provided a variable time between 0 to $5 \mathrm{~ms}$. This made the protocol spontaneous and attacker cannot determine the duration of the CTS timer, thus making it less susceptible to attack and even better than SIGF in the case of CTS rushing attack (Hanapi et al., 2009). However its behavior is non-predictable and in some instances the time might not be enough to favor replies to ORTS signal which subsequently result in retransmission. Retransmission result in increased overhead and energy consumption.

DWSIGF and SIGF also consider distance in choosing a forwarding node. When a node is selected greedily (node that is closest to destination but within the $60^{\circ}$ sextant) that is considered a priority selection while when the choice of the forwarding node falls randomly on a node within the 600 sextant, it is considered a random selection.

These choices of selection are considered to be the variant of the protocol. The diagram (Fig. 1) describes the whole selection process.

\subsection{Energy Consumption}

The communication energy is defined as the sum of the energy required to transmit data, using a transceiver (radio) and the energy required for the data processing to perform encoding and decoding (Pantazis and Vergados, 2007; Heinzelman et al., 1999). Current simulators do not automatically measure energy consumption, leaving it up to the protocol designer to explicitly write code to account for it (Carvalho et al., 2004; Margi and Obraczka, 2004). The development in energy consumption in WSN has been a central focus to most protocol developers since it is considered vital to the communication process. However, this is hindered due to the slow development in battery technology deployed in motes.

Heinzelman et al. (2000) explains radio characteristics of nodes such as the receive, transmit, sleep and ideal states which have greatly contributed to the on-going research on WSN in the area of low-energy (Fig. 2). Various assumptions have been made to determine energy consumptions using the radio characteristics and other researchers have resorted to the use of hardware characteristics specific to a mote. (Sinha et al., 2001; Ye et al., 2002) explores all part of a sensor node to obtain accurate measurement towards energy consumption, showing that all layers of the system including the algorithms, OS and network protocol can adapt to minimize energy usage. However the research was mote specific and does not analyze in part the energy consumed during the communication process. Ngyuyen et al. (2011) used various commercial batteries as a function of different combination of parameters. Communication which is an essential part of the test was seldomly worked on. Simulation studies shows promising result for the assumptions made but most of the assumptions have not been verified against the behavior of a physical radio or mote.

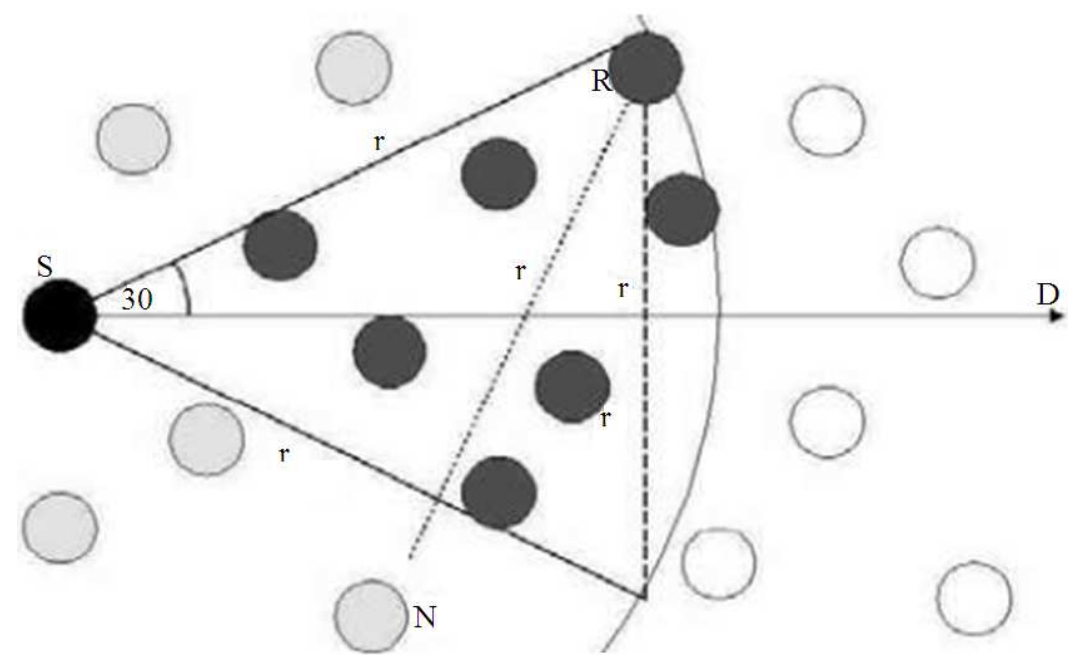

Fig. 1. Forwarding area, $60^{\circ}$ sextant centered on the direct line with the destination source: Hanapi et al. (2009) 


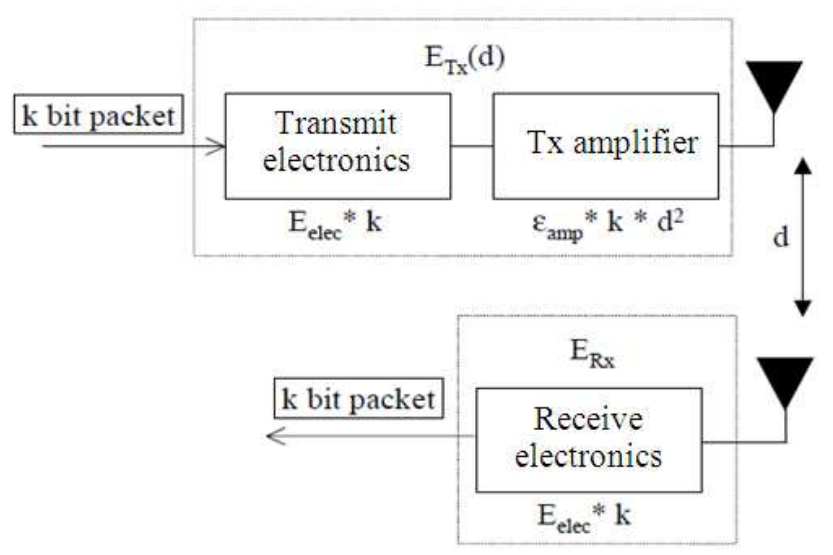

Fig. 2. First order radio model source: Heinzelman et al. (2000)

\section{MATERIALS AND METHODS}

The first order radio model proposed by Heinzelman et al. (2000) provides a starting point for analysis considering its simplicity and flexibility to be adopted in both a single hop network and a multi-hop network Equation 1-3:

Total Transmit $=\mathrm{n}:\left(\mathrm{E}_{\text {Elect }} * \mathrm{k}+\in \operatorname{amp} * \mathrm{k} * \mathrm{r}^{2}\right)$

Total Receive $=(\mathrm{n}-1) * \mathrm{E}_{\text {Elect }} * \mathrm{k}$

Total Energy Dissipated $=\mathrm{k}\left((2 \mathrm{n}-1) \mathrm{E}_{\text {Elect }}+\in \mathrm{amp} n r^{2}\right)$

Our work focuses on multi-hop network where intermediate nodes act as routers and it involves a series of handshaking signals been transmitted and analyzed. The energy for each node that partakes in the event is measured up and analyzed. Thus one can port, for each defined node the equation for the radio modes and calculate the energy depending on the number of times it partakes in each event (radio modes) in the communication process.

In DWSIGF priority and SIGF priority, a greedy algorithm is used in the selection of a CTS sender which responds to a broadcast signal (ORTS signal) after a CTS response time has elapsed. The signal chosen is the one that is closest to the Destination. While In DWSIGF random and SIGF random, any node within the sextant is chosen as the CTS sender (randomly). Node can be immediate neighbor or it can be the node closest to the destination. This can cause an unpredictable behavior in the number of hops before a node reaches its destination.

\subsection{Simulation}

\subsubsection{Assumptions}

In the simulation, nodes were considered to be static once deployed and location aware (based on the GPS reading or any other localization techniques). All nodes had an initial energy of 0.5 joules (each) throughout the network and only transmit and receive radio modes where considered. Since the Energy model (first order radio) provides a commonly used starting point, there is need for specific mote values (voltage or current values). Thus all other assumption as specified by the first order radio model was considered.

\subsubsection{System Configuration}

MATLAB 7.0 was used for the implementation. DWSIGF and SIGF were set to follow the IEEE 802.11 DCF handshaking process. The general simulation parameters are listed in the Table $\mathbf{1 .}$

The simulation was run within a terrain of $150 \times 150$ $m$ with the number of 196 nodes uniformly spread across the terrain, having a communication range $40 \mathrm{~m}$ radius. The result is a mean of hundred simulation runs, it tests many to many CBR flows. Result obtained was based on many to much traffic with six senders situated at the left side of the region and two receivers at the right of the region. The simulation evaluated the protocols (DWSIGF and SIGF) under increasing traffic loads until the traffic becomes 10 packets per second. Energy values are deduced after the data from the simulation was captured. 
Table 1. Simulation parameters

\begin{tabular}{ll}
\hline Terrain & $150 \times 150 \mathrm{~m}$ \\
\hline Number of nodes & 196 \\
Radio Range & $40 \mathrm{~m}$ \\
Application streams & CBR \\
Radio bandwidth & $200 \mathrm{kbps}$ \\
Payload size & 32 bytes \\
CTS packet size & 14 bytes \\
ORTS packet size & 20 bytes \\
ACK packet size & 14 bytes \\
Traffic load & 1 to 10 (Packet/sec) \\
Simulation length & 100 packet, 100 runs \\
\hline
\end{tabular}

\section{RESULTS}

The total energy consumed in the network was measure as traffic was increased. Total energy consumed as described in this research is the sum of energy dissipated by nodes partaking (within the 196 nodes on the terrain) in from when a communication is process is initiated (handshake) to the end of the communication process. This means that not only a single link of the communication process was monitored, but all the nodes in the network which in one way or the other were involved in the communication process. This was necessary because of the ORTS broadcast signal sent to nodes, so one can account for those nodes whose energy were lost in receiving a broadcast but were not chosen as the links for the communication process.

\section{DISCUSSION}

\subsection{The DWSIGF and SIGF Variants}

Figure 3 represents the DWSIGF variants (DWSIGF random and priority) for the energy consumption behavior of both variants. DWSIGF priority shows a consistent behavior in energy consumption as the number of packets/sec is increased. This behavior is due to the stateless behavior of the protocol which tries to evenly distribute communication among all nodes and the greedy algorithm which continuously measures a distance which is almost exactly the same as the node transverses the network from one hop to the other. Even though the window here is dynamic, the risk of retransmission is very small as all nodes which a CTS signal are to be selected can possibly be predicted (at the edge of the range). As the trend increases in pattern continues to widen between the two protocols, an overall difference of about $51.65 \%$ can be observed as the traffic is increased. The increasing energy in DWSIGF random is as a result of the retransmission which occurs and continues to increase in occurrence as the traffic is increased.
In Fig. 4 of the SIGF variants, the behavior pattern for both protocol seems to be consistent. It can be observed that the SIGF random did not increase as the traffic is increased, this is because in SIGF the possibility for the occurrence of retransmission is quite small as a result of the fixed CTS collection window time. SIGF random is still greater than SIGF priority by $29.28 \%$ because of the variation in distance as compared to one which distance is almost the same throughout.

\subsection{Comparing the Variants}

Figure 5 shows the comparison between DWSIGF and SIGF random. Observations show a similar behavior at early stages of the simulation. As the traffic gets more and more saturated, there is an increased chance for the occurrence of retransmission, which causes increase in the energy consumption of DWSIGF. As the traffic is (in packet/sec) is increased, a $16.70 \%$ difference is observed between the two protocols. Selection by priority is described as a way of choosing the node that makes the most progress toward the ultimate destination of the message this method however reduces path dilation, for this reason the chances for occurrence of retransmission in DWSIGF priority is at its bear minimum. A $0.5 \%$ difference is observed in the two protocols (DWSIGF and SIGF) in Fig. 6 for total energy consumed.

The performance of random selection is described as one which suffers since it exhibit erratic behavior in moving message towards the destination. This erratic behavior results in an overall difference in energy consumption of up to $40.44 \%$ between the variant protocols that is random DWSIGF and SIGF and priority DWSIGF and SIGF.

\subsection{Experimental Inferences}

In DWSIGF and SIGF priority, the method used in node selection for message propagation is done in such a way that only the nodes which makes the most progress toward the ultimate destination are selected, typical behavior of a node approaching a base station and similar to the way the greedy algorithm works (Roychowdhury and Patra, 2010). Heinzelman et al. (2000) considers this method as a direct method of transmitting data and infers that it will consume large amount of Tx Power thereby draining the nodes battery and eventually reducing the systems lifetime. However our network is setup in such a way that several hops are made using a prioritized mode of transmission. Total energy seems to be kept at a bare minimum considering the distance the message transverses before reaching the final destination. Inferences made by Heinzelman et al. (2000) on direct method being the optimal will prove positive to our experiment as it is the most acceptable means for data transmission considering Fig. 6. 
Abubakar Idris Umar and Zurina Mohd Hanapi / Journal of Computer Science 10 (9): 1600-1607, 2014

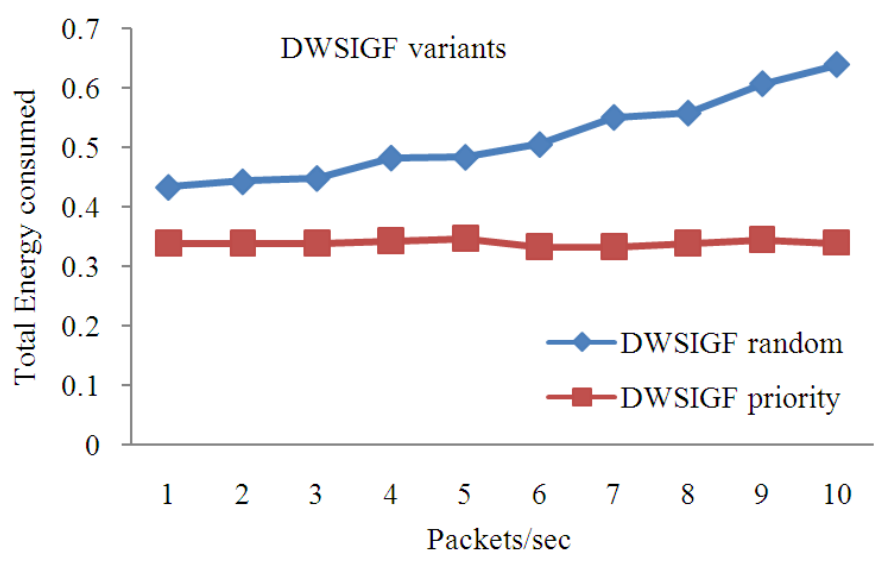

Fig. 3. DWSIGF variants on total energy consumed

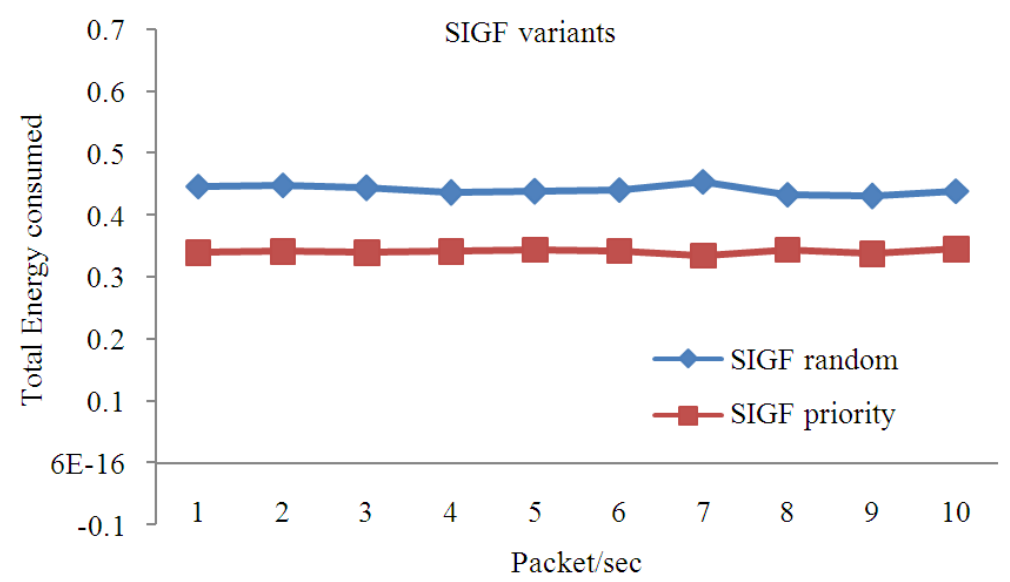

Fig. 4. SIGF variants on total energy consumed

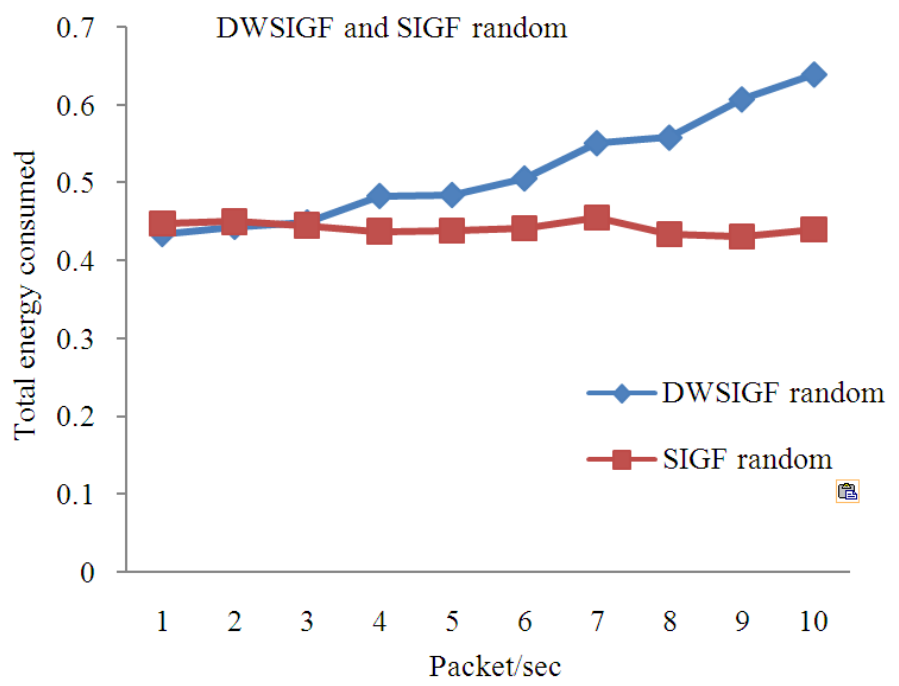

Fig. 5. DWSIGF and SIGF random on total energy consumed 


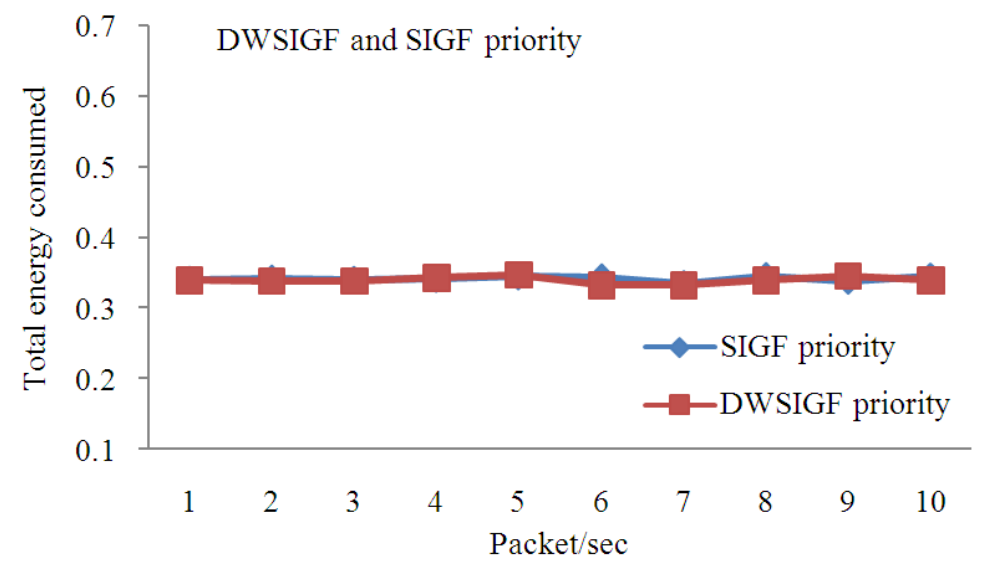

Fig. 6. DWSIGF and SIGF Priority on total energy consumed

However DWSIGF and SIGF random when propagating their messages, the total energy consumed tend to cost more as the data transmitted will have to transverse unknown number of $\mathrm{n}$ nodes in an erratic manner and also the random CTS collection window time which creates retransmission of lost packet. These also agrees with the inferences made by Heinzelman et al. (2000) as observed in Fig. 5.

Considering the shortcomings of energy model (for power aware protocols) in existence (Margi and Obraczka, 2004) our experiment plugged in the first order radio model (Heinzelman et al., 2000) into a stateless routing protocol (Blum et al., 2003; Wood et al., 2006; Hanapi et al., 2009) though the model only considers two radio states (Tx and Rx). It still captures the energy behavior and differences in the two novel protocols. This method of comparison can be considered suitable for determining energy consumption between the two protocols.

\section{CONCLUSION}

The study is limited to DWSIGF and SIGF and only considers the two state of a transceivers (transmit and receive) because in WSN communication, the highest energies are consumed by the Tx and Rx processes of a communication in a node. The priority variant protocols whose operation has been characterize as being dilated when choosing a next hop node, is quite similar to the likes of a direct communication which is also extends to reach a base station. These priority variant protocols (DWSIGF and SIGF) consumed less energy compared to the random variant protocols whose operation has been described as erratic when choosing a next hop node.
Comparison made also infers that DWSIGF which has a variable collection window time suffers from retransmission which occurs from time to time whenever the randomly selected time is not sufficient for a node to receive the required signal, thus consuming more energy than its counterpart (SIGF) which employs a fixed collection window time.

Further work will be done to determine the energy consumed in the entire network while utilizing all radio states and back-off periods. Total data transferred can be captured simultaneously to accurately determine the network lifetime.

\section{REFERENCES}

Akyildiz, I.F., T. Melodia and K.R. Chowdhury, 2007. A survey on wireless multimedia sensor networks. Comput. Netw. 51: 921-960. DOI: 10.1016/j.comnet.2006.10.002

Akyildiz, I.F., W. Su, Y. Sankarasubramaniam and E. Cayirci, 2002. Wireless sensor networks: A survey. Comput. Netw., 38: 393-422. DOI: 10.1016/S13891286(01)00302-4

Al-Karaki, J.N. and A.E. Kamal, 2004. Routing techniques in wireless sensor networks: A survey. IEEE Wireless Commun., 11: 6-28. DOI: 10.1109/MWC.2004.1368893

Blum, B.M., T. He, S. Son and J.A. Stankovic, 2003. IGF: A state-free robust communication protocol for wireless sensor networks. Computer Science Department Technical Report. University of Virginia.

Bulusu, N., J. Heidemann and D. Estrin, 2000. GPS-Less low-cost outdoor localization for very small devices. IEEE Personal Commun., 7: 28-34. DOI: $10.1109 / 98.878533$ 
Carvalho, M.M., C.B. Margi, K. Obraczka and J.J. Garcia-Luna-Aceves, 2004. Modeling energy consumption in single-hop IEEE 802.11 ad hoc networks. Proceedings of the 13th International Conference Computer Communication Networks, Oct. 11-13, IEEE Xplore Press, Chicago, IL., pp: 367-372. DOI: 10.1109/ICCCN.2004.1401671

Gomez, J. A.T. Campbell, M. Naghsineh and C. Bisdikian, 1999. Wireless packet networks. IEEE Trans. Commun.

Hanapi, Z. M., M. Ismail, K. Jumari and M. Mahdavi, 2009. Dynamic window secured implicit geographic forwarding for wireless sensor network. Int. J. Electr. Comput. Eng., 4: 173-179.

He, T., B.M. Blum, Q. Cao, J.A. Stankovic and S.H. Son et al., 2007. Robust and timely communication over highly dynamic sensor networks. Real-Time Syst., 37: 261-289. DOI: 10.1007/s11241-007-9025-2

Heinzelman, W.R., A. Chandrakasan and H. Balakrishnan, 2000. Energy-efficient communication protocol for wireless microsensor networks. Proceedings of the 33rd Hawaii International Conference System Sciences, Jan. 4-7, IEEE Xplore Press, Hawaii, pp: 1-10. DOI: 10.1109/HICSS.2000.926982

Heinzelman, W.R., J. Kulik and H. Balakrishnan, 1999. Adaptive protocols for information dissemination in wireless sensor networks. Proceedings of the 5th Annual ACM/IEEE International Conference Mobile Computing Networking, Aug. 15-19, Seattle, WA., pp: 174-185. DOI:10.1145/313451.313529

Lee, T., C. Qiao, M. Demirbas and J. Xu, 2008. ABC: A simple geographic forwarding scheme capable of bypassing routing holes in sensor networks. Proceedings of 17 th International Conference on Computer Communications and Networks, Aug. 37, IEEE Xplore Press, St. Thomas, US Virgin Islands, pp: 1-8. DOI: 10.1109/ICCCN.2008.ECP.73

Margi, C.B. and K. Obraczka, 2004. Instrumenting network simulators for evaluating energy consumption in power-aware ad-hoc network protocols. Proceedings of the IEEE Computer Society's 12th Annual International Symposium Modeling Analysis Simulation Computer Telecommunications Systems, Oct. 4-8, IEEE Xplore Press, pp: 337-346. DOI: 10.1109/MASCOT.2004.1348288
Ngyuyen, H.A., A. Forster, D. Puccinelli and S. Giordano, 2011. Sensor node lifetime: An experimental study. Proceedings of the IEEE International Conference Pervasive Computing Communications Workshops, Mar. 21-25, IEEE Xplore Press, Seattle, WA., pp: 202-207. DOI: 10.1109/PERCOMW.2011.5766869

Pantazis, N.A and D.D Vergados, 2007. A survey on power control issues in wireless sensor networks. Proceedings of the IEEE Communications Survey Tutorials, (CST' 07), Piscataway, NJ., pp: 86-107. DOI: 10.1109/COMST.2007.4444752

Roychowdhury, S. and C. Patra, 2010. Geographic adaptive fidelity and geographic energy aware routing in ad hoc routing. IJCCT.

Sinha, M.A., A. Wang, A. Chandrakasan, E. Shih and S.-H. Cho, 2001. Physical layer driven protocol and algorithm design for energy-efficient wireless sensor networks. Proceedings of the 7th Annual International Conference Mobile Computing Networking, Jul. 16-21, Italy, pp: 272-287. DOI:10.1145/381677.381703

Stankovic, J.A., 2004. Research challenges for wireless sensor networks. ACM SIGBED Rev. Special Embedded Sen., 1: 9-12. DOI: 10.1145/1121776.1121780

Stankovic, J.A., 2006. Wireless Sensor Networks Technical Report, Department of Computer Science University of Virginia Charlottesville, Virginia.

Wood, A.D, L. Fang, J.A. Stankovic and T. He, 2006. SIGF: A family of configurable, secure routing protocols for wireless sensor networks. Proceeding of the 4th ACM Workshop Security Ad Hoc Sensor Network, (HSN' 06), Alexandria VA., pp: 35-48.

Ye, W., J. Heidemann and D. Estrin, 2002. An energyefficient mac protocol for wireless sensor networks. Proceedings of the 21st Annual Joint Conference Computer Communications Societies, (CCS' 02), IEEE Xplore Press, pp: 1567-1576. DOI: 10.1109/INFCOM.2002.1019408 